\title{
Untersuchungen über die amöboide Glia und Clasmatodendrose.
}

\author{
Von \\ Prof. F. K. Walter. \\ (Aus der Psychiatrischen Klinik Rostock-Gehlsheim \\ [Direktor: Prof. Dr. Rosenfeld].) \\ Mit 9 Textabbildungen.
}

(Eingegangen am 4. Januar 1921.)

In Bd. 47 dieser Zeitschrift berichtete ich über das Vorkommen ausgedehnten Gliazerfalls im Sinne der Cajalschen Clasmatodendrose bei den verschiedensten Psychosen. Anfangs war ich der t'berzeugung, daß dieser Zerfallsprozeß sich im Verlauf längerer Zeit intra vitam entwickele. Weitere Untersuchungen zeigten aber, daß es sich um eine offenbar sehr schnell eintretende Veränderung der Glia handelt, 'bei der agonale und postmortale Einflüsse eine wichtige Rolle spielen.

Die Frage nach dem Alter jeweilig gefundener histologischer Veränderungen ist $\mathrm{ja}$ besonders beim Nervensystem oft außerordentlich schwierig und vielleicht noch wichtiger als in der übrigen Pathologie, da die meist lange Dauer der Psychosen einen entsprechend chronischen Gehirnprozeß voraussetzen, falls man letzteren mit der Genese jener in ursächliche Beziehung bringen will. Die große Bedeutung, die man nach dem heutigen Stand unserer Kenntnisse der Neuroglia zuerkennen muß, fordert daher unbedingt die Beantwortung der Frage: Welche Veränderungen an ihr sind chronische oder akute intravitale Vorgänge, und welche müssen als agonal oder postmortal entstanden angesehen werden?

Ich hatte meine Untersuchungen mit der in der erwähnten Arbeit mitgeteilten Solargylmethode begonnen, deren Resultate in färberischer Hinsicht sich weitgehend mit der Cajalschen Sublimat-Gold-Methode decken, trotzdem die Fixierung des Materials in Alkohol geschah. In einer Reihe von Fällen konnte ich Parallelfärbungen mit beiden Methoden machen, bis dann leider die Unmöglichkeit, brauchbares Solargyl zu bekommen, mich zwang, auf die weitere Anwendung dieses Silbereiweißpräparates zu verzichten ${ }^{1}$ ).

1) Alle Versuche, von der Herstellerin des Solargyls, der schweizerischen Firma Bürgy \& Co. ein gleich brauchbares Präparat wie anfangs zu erhalten, sind bisher leider fruchtlos geblieben; weshalb ich vorläufig von seiner Anwendung abraten muß. 
Cajal hat 1914 in einer eingehenden Arbeit, die mir im Original leider nicht zugänglich war, seine Befunde' und Erfahrungen mit der Sublimat-Gold-Methode bekanntgegeben: Die Struktur der p'asmatischen Glia ist nach ihm schwammartig, wobei die kleinen Lücken wahrscheinlich das Negativ der als G'iosomen und Mitochondrien beschriebenen Strukturen darstellen. Die Ränder der Fortsätze zeigen ähnliche Dornen, wie sie die Golgimethode darstellt. Unter den degenerativen Veränderungen beschreibt er besonders die Zerstückelung der Gliazellen, die er als Clasmatodendrose bezeichnet. Diese kommt, wie Cajal besonders betont, nicht nur als Ausdruck eines pathologischen Prozesses, sondern auch infolge postmortaler Autolyse und bei ungenügender Fixierung vor. Einen sehr wesentlichen Einfluß soll auch die den Tod herbeiführende Erkrankung haben.

Nach Achucarro und Gayarre, die sich besonders mit diesem Prozeß beschäftigt haben, beginnt die Clasmatodendrose damit, daß die Dendriten ungleichmäßige Konturen annehmen, indem sie stellenweise sich teils aufblähen, teils verdünnen. An letzteren Stellen geht dann der Zusammenhang verloren. Die Fragmente haben die Formen von kurzen Stümpfen oder Tropfen. Ihre Größe kann sehr verschieden sein und die einer kleinen G'iazelle erreichen. Häufig geht mit der Zerstückelung der Fortsätze eine Schwellung des Zellkörpers einher, wobei seine Färbbarkeit geringer wird. Nach dem gleichen Autor ist die intravita!e Clasmatodendrose von der auto'ytischen (postmortalen) dadurch unterschieden, da $\beta$ bei der ersteren die Bruchstücke der Dendriten reihenförmig angeordnet sind, während sie im zweiten Fall mehr pulverförmig und weniger kräftig erscheinen.

Schaffer weist darauf hin, daß zuerst eine zunehmende grobe Körnelung des gesamten Plasmas eintritt und schließlich nach der Zerstückelung der Fortsätze ein die graue und weiße Substanz durchsetzender Gliadetritus entsteht, welcher dem Präparat das Aussehen verleiht, als wäre er mit verschieden großen unregelmäßigen Partikelchen übersät. Auf die Frage der postmortalen Entstehung des Gliazerfalls geht er nicht ein.

Die engen Beziehungen zwischen der Cajalschen Clasmatodendrose und der amöboiden Glia betonen dann Achucarro und Gayarre, indem sie schreiben: „Mag es sich nun mit dem Mechanismus der Zerstückelung (Fragmentation) verhalten, wie es wolle, Tatsache ist, daß wir sie in unseren Präparaten manchmal mit einer Anschwellung des Zellkörpers und mit einer schwachen Färbung desselben, sowie gelegentlich mit einer Schrumpfung des Kernes, ähnlich der, welche bei der Bildung der amöboiden und präamöboiden Zellen beobachtet wird, zusammentreffen sehen. Die Zerstückelung der Neurogliazellen, welche mit der Erscheinung sehr reichlicher amöboider Zellen und der 
sug. Füllkörperchen zusammentrifft, ist von Alzheimer studiert worden, der zu dem Schluß kam, daß die genannten Körperchen großenteils aus der Auflösung (desintegración) der Neurogliastrukturen herrühren... Dessenungeachtet kann, da die von Alzheimer angewandten Methoden die Verästelungen des Protoplasmas nicht in solcher Ausdehnung färben wie die Goldmethode, die Evidenz, daß die zerstückelten Körner aus der Zerstückelung der Neuroglia hervorgehen, nicht so groß sein, wie sie sich aus unseren Forschungen ergibt. Dieser Prozeß ist sehr häufig, und wie wir erwähnt haben, befällt er eine einzelne Zelle neben andern normalen ... Der Vorgang der Zerstückelung der Gliazellen zeigt sich in unsern Präparaten sehr reichlich und weit mehr, als uns die Forschungen Alzhei mers vermuten lassen können, und seine Bedeutung ist wahrscheinlich wesentlich für die Neubildung der Astrocyten in amöboide Zellen. Der Anteil, den dieser Zerstückelungsprozeß an der Erzeugung der amöboiden Zellen hat, führt dazu, mehr und mehr der Meinung derer zuzuneigen, welche die Ansicht verteidigen, daß viele von den Zellen von Natur degeneriert sind, wie solches auch für gewisse amöboide Zellen Rosental, Papadia, Buscaino und neuerdings Pandolfi gemeint haben."

Bekanntlich hat Alzhei mer in seiner Gliarbëit die Entstehung der amöboiden Gliazellen ursprünglich als einen progressiven Vorgang aufgefaßt, indem er annahm, daß sie sowohl aus neugebildeten Zellen als auch durch Umwandlung anderer Zellen mit und ohne plasmatische Fortsätze entstehen könnten, indem ihr Zelleib hypertrophiert, während die Phase der Rückbildung durch das Auftreten verschiedener Granula und Cystchen im Innern des Plasmas charakterisiert sei. Vielfach gleichzeitig mit den amöboiden Gliazellen fand Alzheimer die „Füllkörperchen“. Seine Beobachtungen sprachen dafür, daß sie jedenfalls zum größten Teil aus dem Zerfall pathologischer Gliastrukturen hervorgehen, indem die Gliafasern mit ihrem umgebenden Plasma aufquellen und dann zerfallen. Daß es sich dabei um keine postmortalen Vorgänge handele, schloß er daraus, daß er Füllkörperchen bei an. Dysenterie erkrankten Affen fand, deren Gehirne lebenswarm fixiert waren.

Weitere Untersuchungen, die zum großen Teil von Alzheimers Schülern stammen, háben zu einer veränderten Auffassung der amöboiden Glia geführt und gezeigt, daß es sich dabei im wesentlichen um eine regressive Veränderung handelt. Aber wenn in diesem Punkt die Meinungen der Autoren auch ziemlich einheitlich sind, so ist die Frage nach ihren Entstehungsbedingungen und der pathologischen Bedeutung noch keineswegs geklärt.

Schon Eisath wies nachdrücklich darauf hin, daß die Darstellbarkeit der pathologischen Glia (er arbeitete mit der von ihm modifizierten 
Malloryfärbung) von vielen noch nicht erkennbaren Vorbedingungen abhängig sei. So versagte z. B. seine Färbung bei vorangegangener Anämie und Stauungserscheinungen meist, während Tod infolge Pneumonie, Carcinomatose, Ulcus ventriculi und Tuberkulose zu keinen nachweisbaren Veränderungen der Darstellbarkeit der Glia führten. Wichtiger für uns ist aber seine Feststellung, daß agonale und postmortale Veränderungen der Glia zu einer amöboiden Umwandlung führen können, und daß die jeweilige histologische Entscheidung, ob vitale oder autolytische Prozesse vorliegen, unmöglich sei. Sowohl Verschwinden der physiologischen Körnchen und Weigertfasern sowie Quellung des Zellplasmas und Undeutlichwerden seiner Grenzen seien beiden gemein. Auch sei es unmöglich, einen bestimmten Zeitpunkt für den Eintritt postmortaler Veränderungen anzugeben, daß sie aus unbekannten Gründen bald früher bald später beginnen. So konnten in einem Fall, der in der heißen Jahreszeit starb, auch 31 Stunden post mortem keine Leichenveränderungen festgestellt werden, und die Färbung der Gliafasern gelang über Erwarten gut, während letztere bekanntlich im allgemeinen frühzeitige Verarbeitung des Materials zur Voraussetzung hat. Eisath erinnert aber daran, daß innerhalb der ersten 20 Stunden p. m. sich noch keine sehr auffallenden Leichenveränderungen vollziehen können, ohne allerdings den Beweis dafür zu bringen. Bei dieser Sachlage verlangt Eisath möglichst frische Konservierung des Untersuchungsmaterials, glaubt aber unter dieser Voraussetzung auch die gefundenen Gliaveränderungen im Sinne der homogenen und amöboiden Umwandlung mit der vorher beobachteten Geistesstörung in Zusammenhang bringen zu können. Freilich schränkt er die Möglichkeit der kausalen Beziehung zwischen histologischem Befund und chronischer Psychose auf die Fälle ein, wo 1. keine körperliche Erkrankung zum Tode geführt hat, die schädigend auf die Glia einzuwirken vermag (Anämie und die mit Hyperämie einhergehenden Erkrankungen, Vitium cordis usw.) und 2. sich unmittelbar vor dem Tode keine schwere, akute Phase der Psychose eingestellt hat (epileptischer Dämmerzustand, Status epilepticus, katatonische Erregung usw.).

Rosental hat sich dann bemüht, experimentell die Entstehungsbedingungen der amöboiden Glia zu studieren. Er benutzte Pikrotoxin, Guanichin, oxalsaures Natron und anaphylaktisch wirkende Substanzen, mit denen er akute, subakute und chronische Vergiftungen hervorrief. Irgendein gesetzmäßiger Zusammenhang zwischen Art der Vergiftung, Auftreten von Krämpfen und Bildung amöboider Gliazellen ließ sich jedoch nicht feststellen. Nach perakutem Verlauf mit Anfällen und nach chronischem konnten sie fehlen oder in geringem oder ausgesprochenem Maße vorhanden sein. Eine ähnliche nicht erklärbare Unregelmäßigkeit zeigten auch gesunde Tiere, die in gleicher Weise 
getötet und verarbeitet waren: bald weitgehender Amöboidismus, bald kaum eine Andeutung davon. Das Fortschreiten des Prozesses nach dem Tode verfolgte Rosental bei 2 Affen, bei sofort und 6 Stunden post mortem entnommenen Stücken des Gehirnes und Rückenmarks in eindeutiger Weise. Um beim Menschen analoge Bedingungen zu schaffen, legte der Autor Gehirnstückchen in zugedeckte und im Dunkeln aufbewahrte Schalen mit Liquor und fixierte sie nach kürzerer und längerer Zeit. In manchen Fällen traten die ersten Veränderungen nach 18 Stunden (einige Male schon früher), meist aber erst nach 24 Stunden auf. Besonders das Auftreten von Methylblaukörnchen in den anfangs homogenen Zelleibern war öfter sehr charakteristisch, besonders in Umgebung der Gefäße. Einzig die fuchsinophilen Granula glaubt Rosental als Ausdruck lediglich vitaler Vorgänge auffassen zu müssen. Die amöboiden Formen, die diese nicht zeigen, werden vom Autor als Produkt einer Nekrobiose angesehen.

Auch Lotmar kommt auf Grund seiner Dysenterieversuche zu dem Ergebnis, daß die amöboide Umwandlung eine Form des Zelltodes darstellt. Bezüglich der großen Vakuolen weist er aber darauf hin, daß sie nicht nur postmortal, wie Rosental meint, sondern auch intravital bei schwerer toxischer Schädigung entstehen können.

Buscain o hält die amöboide Umwandlung für einen rein passiven Vorgang infolge einer Gleichgewichtsstörung zwischen den Kolloiden der Gliazellen und den umgebenden Flüssigkeiten. Er konnte bei Tieren postmortal oder, nachdem er Gehirnstückchen erhöhter Temperatur $\left(37^{\circ}\right)$ oder verschiedenen Reagenzien (Säuren und Alkalien) aussetzte, die gleichen Gliaveränderungen beobachten.

In eingehender Weise hat sich zuletzt Wohlwill mit der Frage der postmortalen Gliaveränderungen und ihren Beziehungen zur amöboiden Glia befaßt. Zuerst ist wichtig, daß er einen Unterschied zwischen Gehirn und Rückenmark fand, indem 5 Gehirne, die bei der Sektion $(18,21,22,32$, und 36 Stunden p. m.) keine amöboiden Gliazellen zeigten, auch in Stücken, die unter mit Liquor getränkten Wattebäuschchen (auf Eis oder bei Zimmertemperatur) aufbewahrt waren, solche vermissen ließen, während sie in entsprechend behandelten Rückenmarkstücken 2 mal unter 3 Fällen auftraten. Daß diese Versuchsanordnung aber keinesfalls die Bedingungen des in loco belassenen Nervensystems wiedergibt, schließt Wohlwill aus der Tatsache, daß er bei sofortiger Fixation in 15 Fällen, wo pathologische Gliaveränderungen nicht zu erwarten waren, solche auch nicht fand, möchte die Sektion nun 15 oder 84 Stunden p. m. stattgefunden haben. Auch Aufbewahrung von Gehirnstücken in verschiedenen Lösungen war ohne Erfolg. Wohlwill untersuchte deshalb ein sehr umfangreiches Material (126 Fälle) aus dem Eppendorfer Pathologischen Institut darauf hin, 
ob und welche Beziehungen zwischen der Entwicklung amöboider Gliazellen und der zwischen Tod und Sektion verstrichenen Zeit und den voraufgegangenen Krankheiten bestehen. Er kommt zu dem Schluß, daß die Sektionszeit keinen nennenswerten Einfluß auf die Entstehung der amöboiden Glia hat, und daß postmortale Veränderungen, wo sie entstehen, sehr schnell auftreten. Für die Annahme der intravitalen Entstehung spricht nach $\mathrm{ihm}$ die Tatsache, daß amöboide Gliazellen vor allem in den Fällen zu finden waren (57\%), die nachweislich auch andere gröbere Läsionen des Zentralnervensystems aufwiesen, während sie in der Vergleichsserie erheblich seltener $(19 \%)$ nachweisbar waren. Indessen, fügt der Autor hinzu, sei ein Beweis auch hiermit nicht gegeben, da ja nach Rosental gerade Hirnerkrankungen die Entstehung postmortaler Veränderungen begünstigen.

Es ist klar, daß die hier aufgeworfenen Fragen neben dem theoretischen ein großes praktisches Interesse haben. Das Vorkommen der amöboiden Umwandlung ist ja schon nach den Angaben Alzheimers recht verbreitet. Es ist aber noch häufiger, als er annahm, wenn die Clasmatodendrose Cajals tatsächlich nichts anderes als eine andere Darstellungsweise dieses Prozesses ist, wie ich nach meinen eigenen Untersuchungen mit Achucarro und Gayarre annehmen muß.

Morphologisch stellt die Clasmatodendrose eine Zerstückelung der Gliazelle in allen ihren Teilen dar. Die ersten Anzeichen dieses Vorgangs bestehen in einer eigentümlichen Veränderung der plasmatischen Fortsätze. Sie sehen wie borkige, knorrige Äste aus, schwellen an einzelnen Stellen ungleichmäßig an und verdünnen sich an anderen. Vielfach tritt schon früh eine Kontinuitätstrennung auf, so daß die einzelnen Stücke in ihrer ursprünglichen Lage nebeneinander liegen. Oft bleiben feinste Verbindungsfäden noch längere Zeit bestehen. Schließlich löst sich aber jeder Zusammenhang, und die aufgequollenen Stücke zerfallen dann wieder in immer kleinere Teile. Je kleiner sie werden, um so gleichmäßiger sind sie in ihrer Gestalt. Während im Beginn längliche, ovale und polymorphe Formen das Gewöhnliche sind, findet sich schließlich ein mehr oder weniger homogen aussehender Körnchendetritus. Die proximalsten Teile der Dendriten bleiben im allgemeinen am längsten erhalten (Abb. 1), aber später greift die Zerstörung auch auf sie und den Zelleib selbst über, wenngleich der letztere noch lange als unförmliches Gebilde bestehen bleiben kann. In den fortgeschrittenen Stadien dieses Prozesses ist das ganze Grundgewebe fast gleichmäßig mit einer körnigen Detritusmasse übersät, und der Nachweis der Zusammengehörigkeit der einzelnen Kugeln und Bröckel mit ihrer Mutterzelle ist dann unmöglich (Abb. 7, 8). Während die Cajalsche Methode die Zelleibreste als mehr oder weniger homogene, meist dunkel gefärbte Klumpen, häufig mit kurzen Fortsatz- 
stummeln darstellt, zeigen sich mit der Solargylfärbung an den entsprechenden Zellen häufig eigenartige Netz- und Gitterstrukturen (Abb. 2, $a-c$ ), ohne daß bisweilen ein Kern oder eine plasmatische Grund-

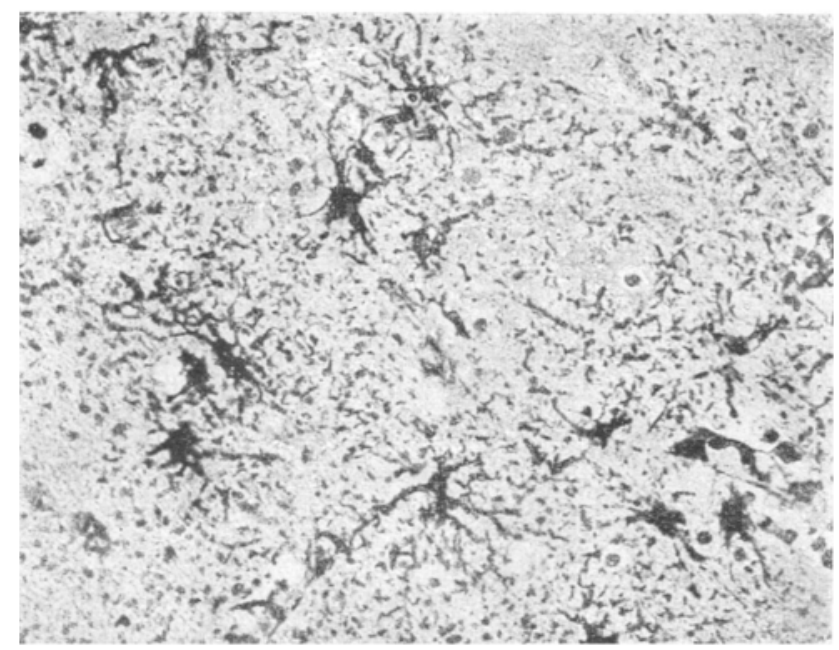

Abb. 1. Aus Markleiste der 1. Frontal-Windung. Die proximalen Teile der Dendriten sind zum Teil nicht kontinuierlich, aber stark varikös verändert. Die Zelleiber zum Teil gequollen. Fall von Dem. paranoides (chroniseher Verlauf), Solargylfärbung. Vergr.: $1: 310$. Mikrophotogramm.
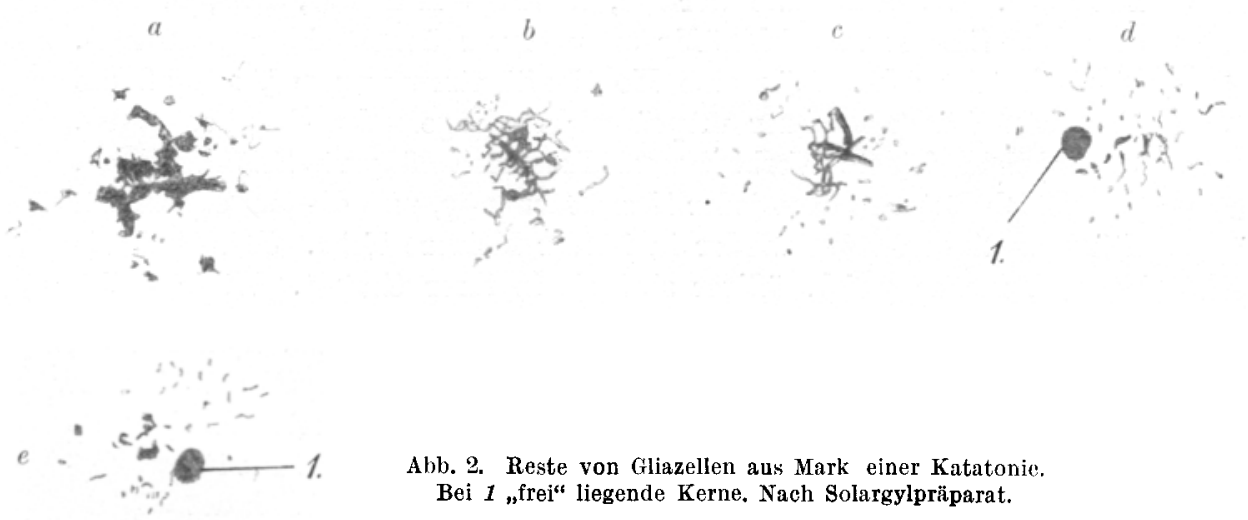

Abb. 2. Reste von Gliazellen aus Mark einer Katatonie. Bei 1 "frei“ liegende Kerne. Nach Solargylpräparat.

substanz sichtbar ist. Manchmal findet sich ein homogen gefärbter Kern zwischen oder neben einem Haufen kleiner Körner oder Faserbruchstücken (Abb. 2, $d-e$ ). Ob es sich dabei um Reste Weigertscher Fasern handelt, kann ich mit Sicherheit nicht sagen.

Daß der Zerfall auch die feinsten plasmatischen Gliafortsätze ergreifen muß, nachdem ihre Loslösung von der zugehörigen Zelle erfolgt 


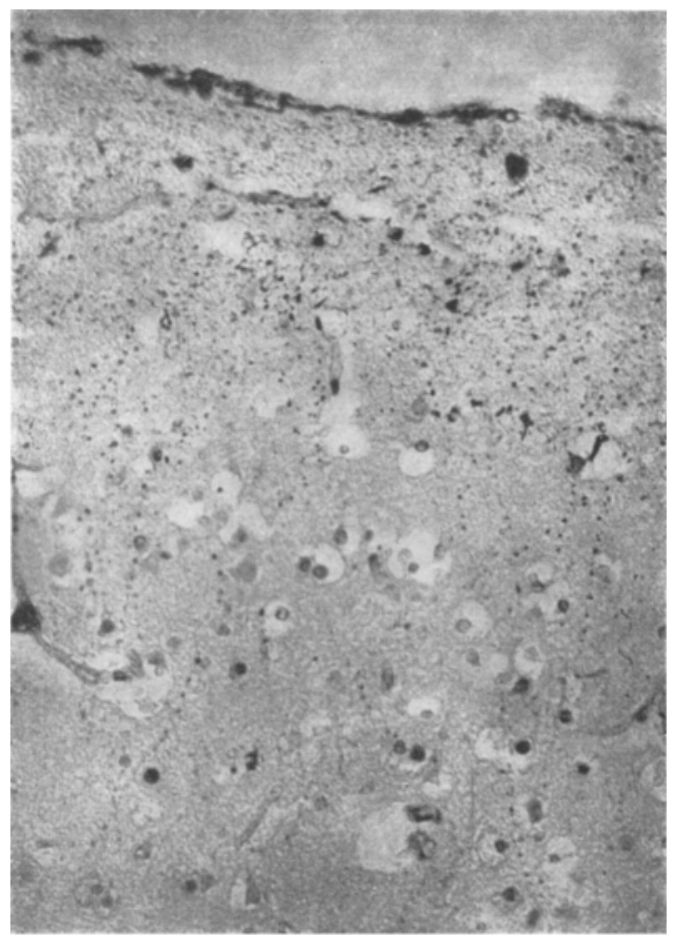

Abb. 3. Calcarinarinde (Fall wie Abb. 1). Von Oberfläche ziehen feine Körnchenreihen in die Rinde herab, die Reste Weigertscher Fasern darstellend. Solargylfärbung. Vergr. 1:220. Mikrophotogramm.

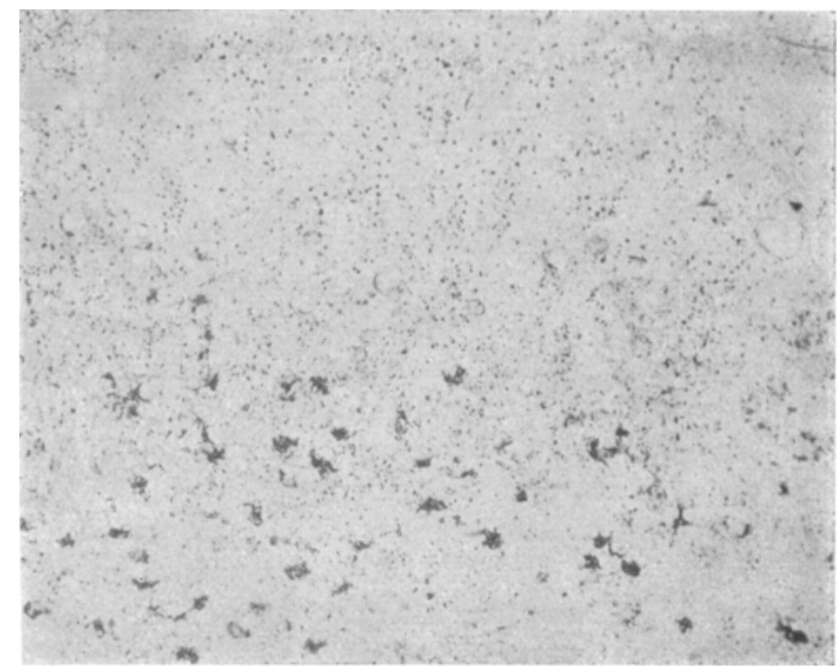

Abb. 4. Ubergang von Mark in Rinde aus Calcarinawindung. (Fall wie Abb. 1 und 2.) Die in die Rinde aufsteigenden Körnerreihen sind zerfallene Weigertsche Fasern. Solargylfärbung. Vergr. $1: 130$. Mikrophotogramm. 
ist, ist ohne weiteres verständlich. Aber auch die Weigertschen Fasern unterliegen dem gleichen körnigen Zerfall. Mit der Solargylmethode könnte ich am Übergang vom Mark zur Rinde oder in den obersten Rindenschichten feinste Körnchenreihen darstellen, die von der Oberfläche herab oder vom Mark in die untersten Rindenschichten hinaufsteigen und in ihrer Verlaufsrichtung und Anordnung so weitgehend den Weigertschen Gliafasern entsprechen, daß ihre Zusammengehörigkeit kaum zweifelhaft erscheint. Abb. 3 und 4 zeigen derartige Bilder aus der Rinde eines Katatonikers. Verfolgt man nun den Prozeß der
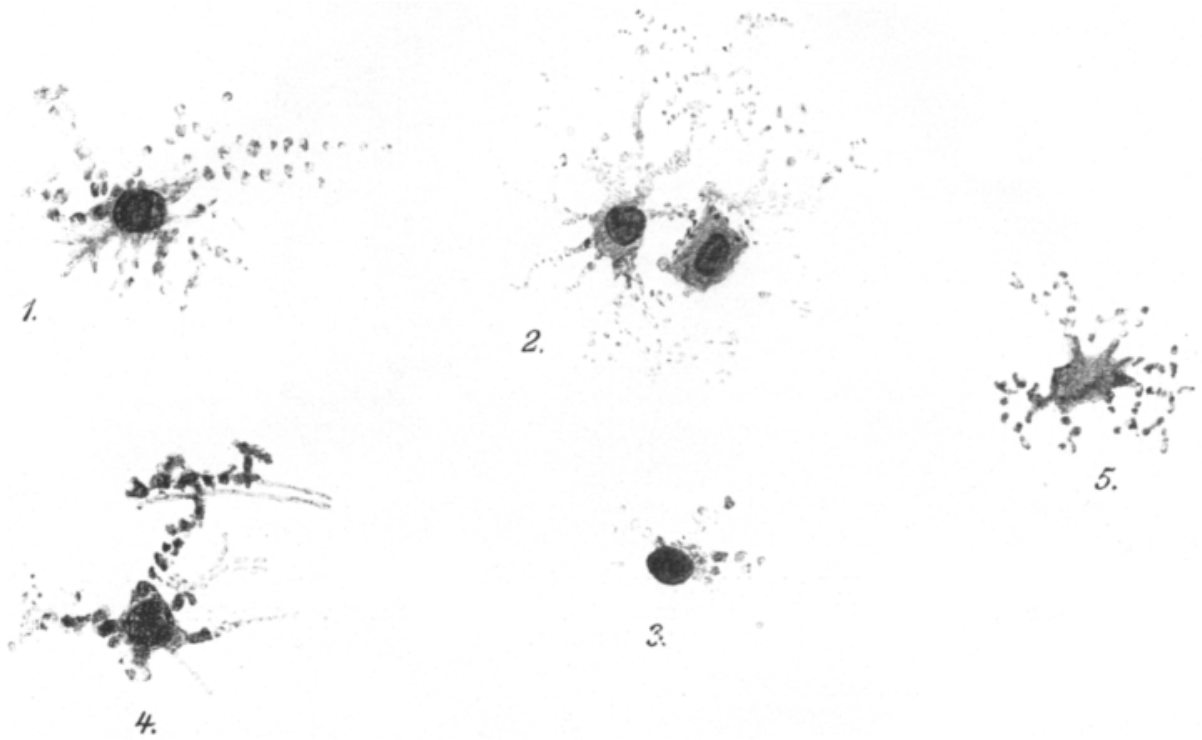

5.

Abb. 5. Einzelne in Zerfall begriffene Gliazellen aus dem tiefen Mark eines Falles von Katatonie. Nach Cajal-Präparat, Lage und Verteilung der Körner gleichen den flbrinoiden und Methylblau-Granula Alzheimers.

einzelnen Zelle genauer, so sieht man sehr oft Bilder, die ohne weiteres an die Abbildungen erinnern, die Alzheimer von amöboiden Gliazellen mit fibrinoiden und Methylblau-Granula gibt (vgl. Tafel 28, Abb. 11, 12, 13 und Tafel 31, Abb. 1-12 der ,Beiträge zur Kenntnis der pathologischen Glia"s usw.). In Abb. 5, 1-5 sind solche einzelnen Zellen mit ihren körnig zerfallenden Dendriten wiedergegeben. Abb. 5, 2 zeigt neben der Gliazelle eine Ganglienzelle, um die sich ein in Auflösung begriffener Fortsatz herumgesponnen hat.

Alzhei mer gewann nicht den Eindruck, daß die erwähnten Granula frei, ohne Beziehungen zu Zellen vorkommen. Wenn er auch meist kein Plasma nachweisen konnte, in dem sie lagen, hielt er sie doch für eingelagerte Stoffwechselprodukte. Die Möglichkeit, ihre Entstehung 
mit den hier angewandten Methoden zu verfolgen, läßt aber kaum eine andere Deutung zu, als daß sie durch Zerstückelung der Plasmafortsätze entstehen. Für diese Deutung sprechen vor allem die erwähnten, anfangs häufig nachweisbaren zarten Verbindungsfäden zwischen einzelnen größeren Zerfallsstücken. Wenn danach die Körner als von ihrem Zelleib völlig losgelöst angesehen werden müssen, so erhebt sich doch die Frage, welche Beziehungen sie zu dem Heldschen Plasmasyncytium haben. Mit der Solargylmethode läßt sich dieses häufig im Mark recht deutlich darstellen, und man kann dann erkennen, daß die Zerfallsprodukte immer innerhalb der Netzbalkengelegensind. Abb. 6 zeigt einen Schnitt aus dem Mark einer senilen Demenz mit fortgeschrittener Clasmatodendrose. Die Lagerung der Bröckel ist so identisch mit dem Verlauf der Netzbalken, daß das Bild nur die eine Deutung zuläßt, daß nämlich das spongiöse Syncytium nicht identisch mit den plasmatischen Gliafortsätzen ist, letztere vielmehr in jenes eingebettet sind, wie das ja besonders Alzheimer immer wieder betont hat. Das Syn-

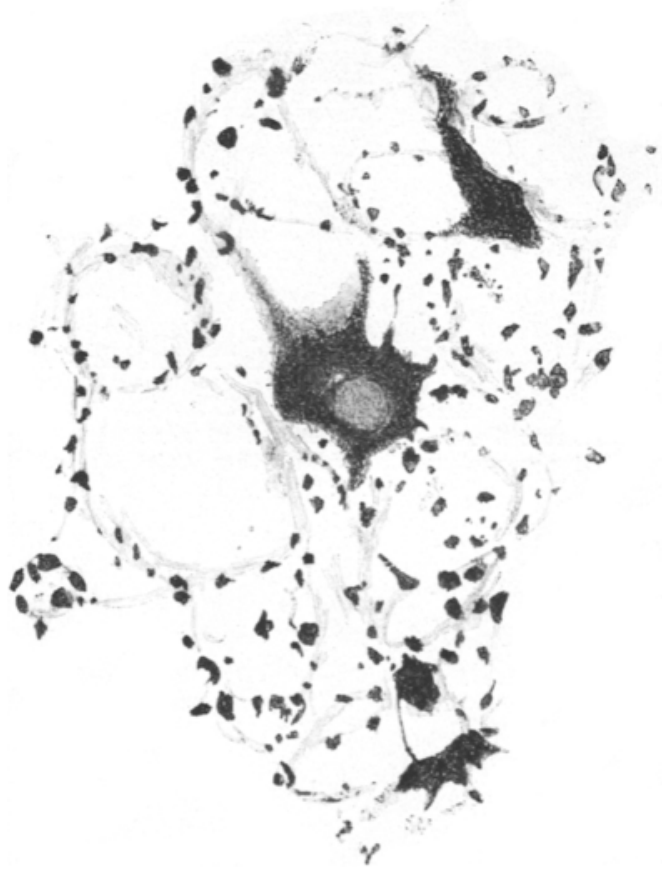

Abb.6. Aus Parietalmark einer senilen Demenz. Die Detrituskörner liegen in den Netzbalken des Heldschen Glia-Syncytiums. Nach Solargylpräparat. cytium bleibt offenbar auch bei völligem Zerfall der Gliazellen in seiner Kontinuität bestehen.

Naturgemäß müssen auch die Gefäßfortsätze der Gliazellen mit ihren Endfüßen dem gleichen Degenerationsprozeß anheimfallen; und es ist leicht, sich von dieser Tatsache zu überzeugen, da jene sich mit den Methoden meist besonders deutlich darstellen lassen. Abb. 7 u. 8 zeigen die Endstadien dieses Prozesses. Besonders auf Abb. 8 erscheinen die Capillaren von einer körnigen, bröckligen Masse eingescheidet. Allerdings findet man bezüglich der Massigkeit dieser Strukturen erhebliche Differenzen, so daß manchmal trotz zahlreicher Detritusbröckel im Grundgewebe die Gefäße kaum hervortreten, während in andern Fällen 


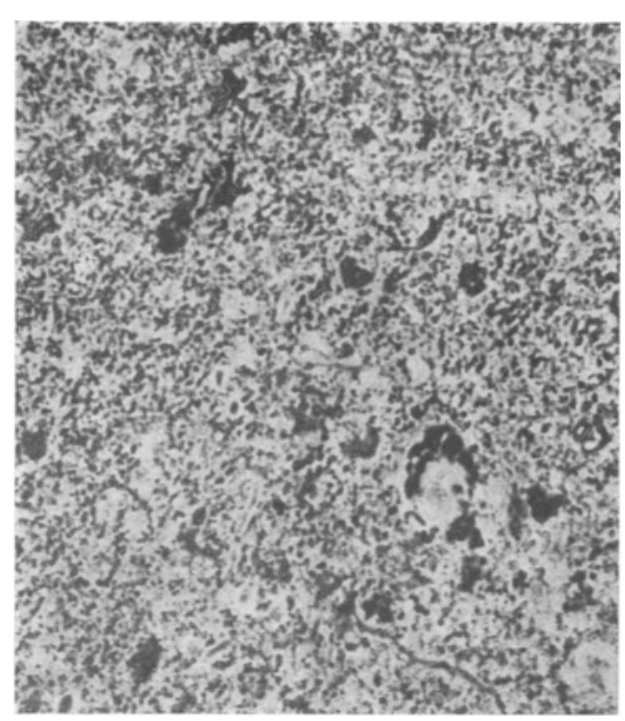

Abb. 7. Tiefes Parietalmark einer Katatonie. Fortgeschrittener Zeriall. Die Zelleiber sind nur noch als unförmige, gequollene Klımpen sichtbar. Cajalfärbung. Vergr. 1: 290.

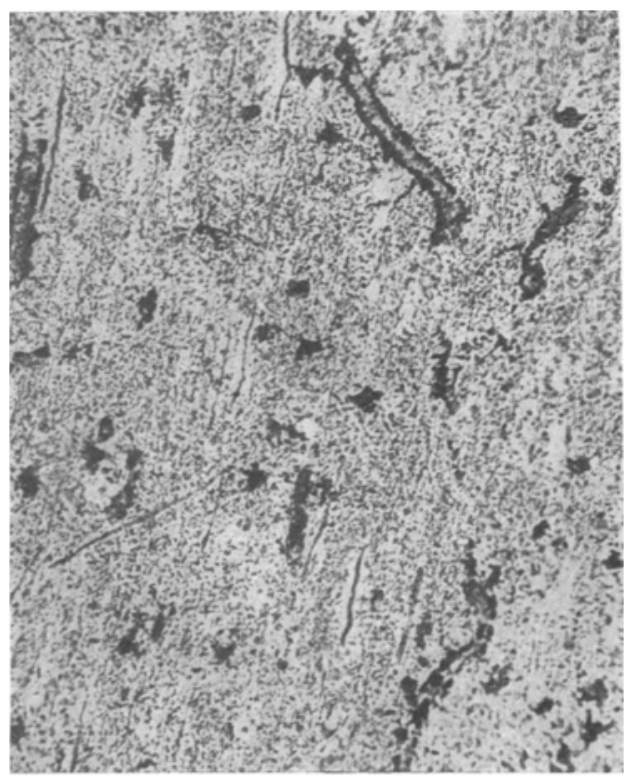

Abb. 8. Parietalmark eines Falles von Katatonie mit fortgeschrittenem Gliazerfall und Anhäufung der Detrituskörner um die Capillaren. Cajalfärbung. Vergr. 1 : 130. geradezu eine Anreicherung an diesen Stellen stattgefunden zu haben scheint. $\mathrm{Ob}$ dies auf Verschiedenheiten der Färbung beruht, vermag ich nicht zu sagen. Sicherlich tritt meist eine Schwellung der Endkolben beim Beginn des Zerfalls ein, worauf schon Schaffer hinwies. Eine analoge Volumzunahme ist oft auch an den Zelleibern selbst zu beobachten. Man braucht nur Schnitte zu durchmustern, in denen neben normalen erhaltenen Gliazellen solche mit fortgeschrittener Zerstückelung der Dendriten sich finden. Die ursprünglich konkav begrenzten Zelleiber quellen zugleich mit den ihnen noch anhaftenden kürzeren oder längeren Fortsatzstummeln auf und bilden so Formen, die oft völlig den mit den Alzheimerschen Methoden darstellbaren amöboiden Zellen gleichen (Abb. 7 r. unten und Abb. 8).

Die Färbbarkeit ist in den Anfangsstadien der Clasmatodendrose für beide angewandten Methoden gesteigert. Nur in den fortgeschrittensten Stadien läßt sie nach. Mit der Cajalschen Methode nimmt das Zellplasma dann gewöhnlich einen schmutzig grauschwarzen Ton an, wie das auch Achucarro und Gayarre betonen, 
während im Solargylpräparat die erwähnten faserigen Netzstrukturen oder ganz unscharf begrenzte gelappte plasmatische Zelleiber erscheinen. Ich möchte jedoch darauf hinweisen, daß zu lange Formolfixierung ähnliche Bilder bei der Vergoldung hervorrufen kann.

Um über das Verhältnis zwischen Clasmatodendrose und amöboider Umwandlung der Glia genaueren Aufschluß zu exlangen, habe ich bei einer größeren Anzahl von Gehirnen je 2 aufeinanderfolgende Blöcke mit den verschiedenen Methoden gefärbt. Von den Alzheimerschen Methoden wurde regelmäßig Nr. IV (modifizierte Malloryfärbung) angewandt, gelegentlich auch Nr. V (Mannsche Färbung) und Nr. VI (Fuchsin-Lichtgrün).

Ich lasse die Resultate kürz folgen und bemerke nur, da $B$ bei allen Gehirnen auch die üblichen Nervenzellfärbungen angewandt wurden. Außer dem Alter und der psychiatrischen Diagnose vermerke ich gleich hier die körperliche zum Tode führende Erkrankung und den Zeitpunkt der Sektion, da ich auf ihren etwaigen Zusammenhang mit dem Gliazerfall weiter unten noch eingehe:

1. St., 29 Jahre, ơ. Katatonie. Gest. an Dysenterie 8. VI. 1917. Letzte Temperatur 36,0. Sektion $11^{1 / 2}$ St. p. m. Gehirn ziemlich hyperämisch, sonst o. B.

Cajalfärbung: In gesammter Rinde reichlich Gliabröckel und Körner. Nur in sechster Schicht einige Astrocyten mit erhaltenen Dendriten.

Im obersten Mark reichlich gequollene Zelleiber mit kurzen, dicken Fortsätzen. In tiefem Mark völliger Zerfall der Dendriten und meist auch der Zelleiber. Im Grundgewebe massenhaft Gliadetritus in Form von Körnern und Bröckel.

Malloryfärbung: Neben großen homogenen amöboiden Gliazellen reichlich solche mit Cysten. Starke Anhäufung von Schollen und Füllkörperchen um die Gefäße.

2. Pr., 28 Janre, o. Katatonie (erregte Form). Gest. an Dysenterie den 4. IV. 1918. Kein Fieber. Sektion $16^{1 / 2}$ St. p. m. Gehirn o. B.

Cajalfärbung: In Rinde reichlich Körner, keine erhaltenen Spinnenzellen. Im gesamten Mark, nach Tiefe hin zunehmend, massenhaft Körner und Schollen, um Capillaren vermehrt. Dazwischen Zelleiber mit rudimentären Dendriten, in Form oft sehr an amöboide Zellen erinnernd.

Solargylfärbung: In Rinde keine Spinnenzellen. Im oberflächlichen Mark ziemlich viele unregelmäßige Gitterstrukturen. Das gesamte Grundgewebe ist mit zahlreichen kleinen Bröckeln und Körnern übersät. Um Capillaren Anhäufung.

Malloryfärbung: Zahlreiche, meist homogene amöboide Gliazellen ohne Vakuolen im Mark.

3. N., 45 Jahre, o. Katatone Verblödung, ruhige Form. Gest. 11. XII. 1918 an Pneumonie. Temperatur in letzten Tagen um $39^{\circ}$. Sektion $2 \frac{1}{2}$ St. p. m. Gehirn o. B.

Cajalfärbung: In Molekularschicht vereinzelte zarte Spinnenzellen. In 2. und 3. Schicht bandartige Verbreiterung und grobe Körnelung der Dendriten. In unteren Schichten dichtes Flechtwerk feiner, gut erhaltener plasmatischer Dendriten. Im tiefen Frontalmark zwischen gut erhaltenen Zellen einige Körner. Im tiefen Parietalmark zeigt ein Teil der Dendriten beginnende Clasmatodendrose. Malloryfärbung: Keine amöboiden Gliazellen. 
4. v. O., 64 Jahre, ơ. Inkohärente Verblödung, chronischer Zustand. Gest. 15. XII. 1917 an Dysenterie. Zuletzt subfebrile Temperatur.

Sektion $4^{1 / 2}$ St. p. m. Mäßiges Piaödem, sonst Gehirn o. B.

Cajalfärbung: In 1.-5. Schicht nur Körner und Bröckel, keine Spinnenzellen, in 6. Schicht und oberster Markleiste einige erhaltene Astrocyten (im Frontalteil etwas mehr als im Parietal-, Temporal- und Occipitalteil). Im gesamten Mark nach Tiefe zunehmender Zerfall der Glia und massenhaft Körner im Zwischengewebe.

Solargylfärbung: Keine erhaltenen Spinnenzellen, dagegen im Mark reichlich Zellen mit großem Plasmaleib, lappenförmigen kurzen Fortsätzen und unscharfer Begrenzung.

Malloryfärbung: Zahlreiche amöboide Gliazellen im gesamten Mark mit größeren und kleineren Vałuolen. Reichliche Füllkörperchen.

5. M., 23 Jahre, ơ. Katatonie, chronische iuhige Form. Gest. an Tbc. pulmonum et intest. In letzten Tagen Fieber um $39^{\circ}$. Sektion $5 \frac{1}{2}$ St. p. m. Gehirn o. B.

Cajalfärbung: In allen untersuchten Hirnteilen der gleiche Befund. In Molekularschicht keine Astrocyten gefärbt, auch keine Detrituskörner, in 2.-3. Schicht reichlich Spinnenzellen mit bandartig verdickten und grob gekörnten Fortsätzen. 4.-6. Schicht und das Gyrusmark zeigt dichtes Flechtwerk gut erhaltener Dendriten. Im tiefen Mark Fortsätze z. T. grobkörnig und varikös, aber überall noch kontinuierlich. Dazwischen feine Körner in ziemlich geringer Anzahl.

Malloryfärbung: Keine amöboiden Gliazellen.

6. L., 31 Jahre, 0 . Katatonie, chronische ruhige Form. Gest. an Tbc. pulm. Sektion $18 \frac{1}{2}$ St. p. m. Fieber bis $40^{\circ}$. Am letzten Tag kein Fieber.

Cajalfärbung: In Frontal-, Parietal-, Temporal und Occipitalteil der gleiche Befund: 1. Schicht keine Gliazellen gefärbt, 2.-3. Schicht Spinnenzellen mit bandförmigen, stark gekörnten, z. T. diskontinuierlichen Dendriten, mäßig reichliche Körner im Grundgewebe. In 4.-6. Schicht sowie Markleiste dichtes Geflecht z. T. gut erhaltener, z. T. gekörnter Fortsätze.

In Mark nach Tiefe stark zunehmende Clasmatodendrose und Körner im Zwischengewebe. Keine Anhäufung um Gefäße. Im tiefen Mark keine erhaltene Zelle mehr und zahlreiche Körner und Bröckel.

Malloryfärbung: Im tiefen Mark einzelne homogene Gliazellen einzeln mit Methylblau-Granula.

7. H., 34 Jahre, o. Katatonie, chronische ruhige Form. Gest. 25. I. 1919 an Pneumonie. In letzten Tagen subfebrile Temperaturen, vorher Fieber bis 39,2.

Sektion $1 \frac{1}{2}$ St. p. m. Ziemlich starkes. Piaödem, Gehirn o. B.

Cajalfärbung: In Frontal-, Parietal-, Temporal- und Occipitalteil der gleiche Befund: In l. Schicht zarte, gut erhaltene Spinnenzellen, 2.-3. Schicht: Reichlich Zellen mit bandförmigen, gekörnten Fortsätzen. 4.-6. Schicht: Dichtes, gut erhaltenes Flechtwerk plasmatischer Fasern, keine Körner, ebenso Gyrusmark. Im tiefen Mark und Corp. striatum sind Dendriten, z. T. grobkörnig und finden sich mäßig zahlreiche Körner im Zwischengewebe.

Malloryfärbung: Keine amöboiden Gliazellen.

8. M., 54 Jahre, ㅇ․ Hebephrene Verblödung. Gest. 5. VI. 1919 an Sepsis und Dysenterie. Fieber zwischen 38 und $39^{\circ}$. Sektion $1 \frac{1}{2}$ St. p. m. Ziemlich reichliches Piaödem, sonst Gehirn o. B.

Cajalfärbung: In 1. und 2. Schicht Spinnenzellen mit gekörnten Fortsätzen, im tiefen Mark einzelne variköse Dendriten und Körner im Zwischengewebe, sonst plasmatische Glia überall gut erhalten.

Malloryfärbung: Keine amöboiden Zellen. 
9. B., 43 Jahre, ㅇ. Hebephrene Verblödung. Gest. 10. II. 1919 an Lungenabsceß und Pleuritis mit Sepsis. Temperatur $38-40^{\circ}$.

Sektion $4^{1} / 2$ St. p. m. Gehirn o. B.

Cajalfärbung: Befund in allen Teilen gleich. In 1.-3. Schicht leichte Hypertrophie der Astrocyten mit gekörnten und z. T. diskontinuierlichen Fortsätzen. In 4.-6. Schicht und Markleiste gut erhaltene Astrocyten.

Im Mark nach Tiefe stark zunehmender Zerfall. Im tiefen Mark fast keine erhaltene Zelle mehr und reichliche Detrituskörner.

Im Corpus striatum nur geringer Zerfall.

Malloryfärbung: Im tiefen Mark ziemlich reichlich amöboide Zellen mit teils homogenem, teils gekörntem Plasma.

10. G., 26 Jahre, Ơ. Katatonischer Stupor. Gest. 19. II. 1917 an Tbc. pulm. Temperatur zwischen 38 u. $39^{\circ}$. Sektion $4^{1 / 2}$ St. p. m. Gehirn o. B.

Cajalfärbung: In allen Teilen gleicher Befund. 1.-5. Schicht zeigt nur einzelne Körner, Spinnenzellen sind nicht gefärbt. In 6. Schicht und Gyrusmark gut erhaltene Glia. Im tiefen Mark beginnender Zerfall, Fortsätze meist noch kontinuierlich.

Malloryfärbung: Keine amöboiden Zellen.

11. B., 45 Jahre, $ণ$. Katatonie, erregte Form, gest. an Miliartuberkulose. Temperatur zwischen 37 u. $38^{\circ}$. Sektion $12^{1 / 2}$ St. p. m. Gehirn o. B.

Cajalfärbung: In allen Teilen gleicher Befund.

In Rinde nur reichlich Körner und Bröckel besonders um Gefäße. In Markleiste noch einige leidlich erhaltene Astrocyten. Im gesamten Mark massenhaft Körner und Bröckel, die förmliche Krusten um Capillaren bilden (Abb. 8). Zellleiber klumpig, z. T. mit kurzen, dicken Fortsätzen, z. T. völlig zerfallen. Ebenso in Stammganglien. In innerer Kapsel sind Zellen besser erhalten.

Malloryfärbung: Zahlreiche homogene und gekörnte amöboide Zellen.

12. W., 52 Jahre, $\Varangle$. Paranoide Demenz, gest. an perniziöser Anämie. Keine Temperatur. Sektion $71 / 2$ St. p. m. Starke Anämie des Gehirns, kleinste capilläre Blutungen, besonders im Balken und Parietale.

Histologisch: Typischer Befund.

Cajalfärbung: In gesamter Rinde erhebliche Hypertrophie der plasmatischen Glia. Nur in 2. Schicht einige bröckelige Fortsätze, sonst gut erhalten. Im Mark nach Tiefe schnell zunehmender Zerfall der Glia. Im tiefen Mark massenhaft Körner und Bröckel im Grundgewebe, um Capillaren vermehrt.

Malloryfärbung: Im Mark einzelne homogene amöboide Zellen únd zahlreiche Zellen mit kleinem, aber stark gekörntem Plasmaleib.

13. Kl., 78 Jahre, o. Senile Demenz. Gest. 5. V. 1919 an akutem Glottisödem. Kein Fieber. Sektion 5 St. p. m. Gehirn atrophisch, ziemlich derbe, keine Herde.

Cajalfärbung: In Rinde nur einige hypertrophische Astrocyten gefärbt. In 2.-3. Schicht sind Dendriten z. 'T. bröckelig, sonst gut erhaltene Fortsätze. Im Gyrusmark Glia gut erhalten. Im tiefen Mark zwischen gut erhaltenen Zellen reichlich Körner und mehr oder weniger stark zerfallene Astrocyten.

Malloryfärbung: Im tiefen Mark ziemlich reichliche, meist stark gekörnte amöboide Zellen.

14. Sch., 80 Jahre, $\subsetneq$. Senile Demenz. Gest. 13. V. 1919 an tuberkulöser Pleuritis. Temperatur 38-39 . Sektion $61 / 2$ St. p. m. Mäßige Hirnatrophie und Arteriosklerose. Keine Herde.

Cajalfärbung: In 1.-3. Schicht einige grobgekörnte Dendriten, sonst Rindenglia überall gut erhalten. Gyrusmark. Glia gut erhalten. Im tiefen Mark zu- 
nehmende Clasmatodendrose mäßigen Grades, ziemlich reichlich Körner im Grundgewebe.

Malloryfärbung: Keine sichern amöboiden Gliazellen.

15. E., 88 Jahre, ㅇ. Arteriosklerotische Demenz. Gest. 9. V. 1919 an Apoplexie. Temperatur bis $38,5^{\circ}$. Sektion $1 \frac{1}{2}$ St. p. m. Starke Arteriosklerose. Frischer Erweichungsherd im 1. Gyrus angularis.

Cajalfärbung: Rindenglia, soweit gefärbt, gut erhalten. Auch im tiefen Mark, kein Zerfall, abgesehen von unmittelbarer Umgebung des Herdes.

Malloryfärbung: Keine amöboiden Zellen.

16. P., 67 Jahre, ㅇ. Seniler Verwirrtheitszustand. Gest. 23. VIII. 1919 an Perikarditis. Sektion 36 St. p. m. Gehirn mäßig injiziert. Mäßige Gefäßsklerose.

Cajalfärbung: 1.-2. Schicht deutliche Hyperplasie der plasmatischen Glia, kein Zerfall. In 3. Schicht nur kleine Körner, keine erhaltenen Astrocyten. Übrige Rinde und oberflächliches Mark zeigt gut erhaltene Glia. In tieferem Mark variköse z. T. schon diskontinuierliche Dendriten.

Malloryfärbung: Keine amöboiden Gliazellen.

17. R., 61 Jahre, o. Paralyse. Verwirrter Erregungszustand. Gest. an Herzlähmung, keine Temperatur. Sektion 6 St. p. m. Starke Leptomeningitis, mäßiger Hydrocephalus.

Cajalfärbung: Erhebliche Hypertrophie der Randglia, geringere der übrigen Rinde. Hier Dendriten, z. T. varikös, aber meist noch kontinuierlich. Im Mark nach Tiefe zunehmender Zerfall mit Bröckel und Körnern im Zwischengewebe.

Malloryfärbung: Im tieferen Mark einzelne amöboide Zellen mit gekörntem Plasmaleib.

18. M., 43 Jahre, o. Paralyse. Gest. 13. VI. 1919 an Pneumonie. Temperatur bis $40^{\circ}$. Sektion 2 St. p. m. Geringe Leptomeningitis, geringer Hydrocephalus.

Cajalfärbung: Deutliche Hypertrophie der Rindenglia. In 3.-4. Schicht Dendriten, z. T. varikös, z. T. diskontinuierlich. Im Mark kein Zerfall.

Malloryfärbung: Keine amöboiden Zellen.

19. Schr., 42 Jahre, ơ. Paralyse. Gest. an Kachexie bei Enteritis. Keine Temperatur. Sektion 5 St. p. m. Hirn anämisch, geringe Leptomeningitis, geringer Hydrocephalus. Starke Ependymitis granularis.

Cajalfärbung: Rindenglia mäBig hypertrophisch. Dendriten grobkörnig in 2. und 3. Schicht einzelne diskontinuierliche Fortsätze, sonst gut erhalten. Im Mark nach Tiefe stark zunehmender Zerfall mit massenhaft Körnern im Zwischengewebe.

Malloryfärbung: Im Mark vereinzelte homogene amöboide Zellen. Viele helle Kerne mit kleinem Plasmaleib.

20. St., 34 Jahre, $\odot$. Landrysche Paralyse. Gest. an Atemlähmung. Kein Fieber. Sektion 16 St. p. m. Gehirn ziemlich blutreich, sonst o. B.

Cajalfärbung: In 1. Schicht gut erhaltene zarte Astrocyten. In 2.-4. Schicht nur Körner, in 5.-6. Schicht einzelne gut erhaltene Zellen, dazwischen Körner. Im Mark nach Tiefe stark zunehmende Clasmatodendrose mit vielen Detrituskörnern im Zwischengewebe.

Malloryfärbung: Mäßig reichliche meist homogene amöboide Glizaellen.

21. P., 62 Jahre, o. Hypophysentumor. Gest. an eitriger Bronchitis mit Herzschwäche. Sektion 3/4 St. p. m. Starkes Piaödem, Hirnsubstanz feucht. Cystischer, in Gyrus orbitalis hineinreichender Hypophysentumor. 
Cajalfärbung: In 2. Rindenschicht sind Dendriten grobkörnig, sonst in Rinde und Mark kein Zerfall, außer in unmittelbarer Umgebung des Tumors.

Malloryfärbung: Keine amöboiden Zellen.

22. 30 Jahre, $\sigma^{\top}$. Amaurotische Idiotie. Gest. an Dysenterie. Kein Fieber. Sektion 32 St. p. m. Erhebliches Piaödem. Pia verdickt.

Cajalfärbung: Nur in Markleiste noch einige Astrocyten mit kontinuierlichen, aber varikösen Dendriten. In Rinde reichlich grobe Körner und Bröckel, die erhaltenen Zelleiber sind deutlich vergrößert. Im gesamten Mark massenhaft Körner und Bröckel, keine erhaltenen Astrocyten.

Malloryfärbung: Zahlreiche amöboide Zellen, teils mit homogenem, teils gekörntem Plasmaleib. Reichlich Füllkörperchen.

23. Sch., 70 Jahre, ㅇ․ Kohlenoxydvergiftung. Gest. im Koma 29. V. 1919. Kurz ante exitum 39,5 Temperatur. Sektion $81 / 2$ St. p. m. Starkes Piaödem. Starke Hyperämie, besonders in Basalganglien. Geringe Arteriosklerose.

Cajalfärbung: In 1.-4. Schicht nur Körner, in 5. Schicht Spinnenzellen mit varikösen Dendriten. In 6. Schicht und Markleiste gut erhaltene Astrocyten. In Mark, nach Tiefe zunehmend, ziemlich starker Zerfall der Glia.

Malloryfärbung: Nicht sehr zahlreiche, stark gekörnte amöboide Gliazellen.

24. Gr., 52 Jahre, ơ. Verwirrter Erregungszustand (Delirium acutum). Gest. an Herzschwäche, kein Fieber. Sektion 1 St. p. m. Pia stark hyperämisch, kein Ödem. Keine Hirnschwellung. Im r. Thalamus kirschkerngroße Cyste mit bräunlicher, glatter Wandung.

Cajalfärbung: Nur in Markleiste gut erhaltene Spinnenzellen, sonst im gesamten Gehirn fortgeschrittener Zerfall mit zahlreichen Körnern im Grundgewebe.

Malloryfärbung: Im Frontalmark mäßig reichliche, meist homogene amöboide Gliazellen. Im Temporalmark noch mehr amöboide Zellen, vielfach mit Lipoidcystchen.

25. M., 57 Jahre, 오. Verwirrtheitszustand (Katatonie? Symptomatische Psychose ?). Gest. an Herzschwäche bei Parotisabsceß. Sektion 3 St. p. m. Mäßiges Piaödem, mäßige Hyperämie.

Cajalfärbung: Nur im tiefen Mark einzelne variköse Dendriten, sonst Glia gut erhalten.

Malloryfärbung: Keine amöboiden Zellen.

Úbersieht man die Fälle im Zusammenhang, so ergibt sich, daß überall da, wo starke Clasmatodendrose vorhanden ist, auch amöboide Zellen nicht fehlen. Differenzen finden sich nur dort, wo der Gliazerfall im Gold- oder Silberpräparat nicht sehr stark ist, besonders in Fall 10, $12,14,16$ und 19. Aber überall kann man sich leicht davon überzeugen, daß es sehr viel einfacher und vollständiger gelingt, die Clasmatodendrose nachzuweisen als das Vorkommen amöboider Zellen. Ich lasse dahingestellt, ob nicht auch dort bei längerem Suchen noch solche gefunden wären, wo die Cajalsche Methode beginnenden Zerfall aufwies. Es ist ja oft keineswegs leicht, zu entscheiden, ob man eine Zelle schon als amöboide ansprechen soll, da alle Ubergänge zur normalen vorkommen.

Das Ergebnis der angeführten Fälle habe ich in einer ganzen Anzahl weiterer, die ich aber einzeln aufzuführen für überflüssig halte, 
bestätigt gefunden. Wenn es auch wünschenswert erscheint, die Homo'ogie der mit den verschiedenen Methoden erhaltenen Bilder noch weiter durchzuführen, so glaube ich doch, daß an der Identität der Clasmatodendrose und amöboiden Umwandlung kein Zweifel bestehen kann.

Für die prinzipielle Auffassung des Prozesses ist nun wichtig, daß er im Gold- oder Silberpräparat sich von vornherein an voll entwickelten Zellen abspielt und schon in den ersten Phasen durchaus degenerativen Charakter trägt. Die mitgeteilten Vergleichsuntersuchungen machen es sehr wahrscheinlich, daß der amöboide Charakter der Zellen erst relativ spät, jedenfalls nach Beginn der Kontinuitätstrennung der Dendriten mit den Alzheimerschen Methoden nachweisbar wird, wenn auch der Beweis hierfür an der einzelnen Zelle aus technischen Gründen z. Zt. nicht zu führen ist.

Es ist klar, daß es unter diesen Umständen näher liegt, die bei der amöboiden Umwandlung nachweisbaren verschiedenen Granula als Zerfallsprodukte der eigenen Zelle anzusehen, als sie auf Assimilation pathologischer Stoffwechselprodukte zurückzuführen. Freilich schließt eins das andre nicht aus, aber es wird sich im folgenden zeigen, daß sehr vieles auf einen primären Gliazerfall hinweist, unabhängig von jeweiligen Erkrankungen der Nervenzellen. Ich möchte aber gleich hier bemerken, daß die Lipoidcystchen wohl nicht auf eine Stufe mit den verschiedenen Granula zu stellen sind, da ich sie bei Anwendung der Cajalschen Methode gar nicht ganz selten auch in ,normalen“, d. h. nicht clasmatodendrotisch veränderten Zellen fand. Wenn Rosental gerade diese als Zeichen postmortaler Veränderungen ansieht, so kann ich ihm darin mit Lotmar nicht beistimmen.

Es lag nahe, mit Hilfe der neuen Methoden den Ursachen des Gliazerfalls erneut nachzugehen. Da mehrfache Beobachtungen auf die Wichtigkeit einer frühzeitigen Sektion hinzuweisen schienen, habe ich ähnlich wie Wohlwill statistische Untersuchungen über die Beziehungen zwischen Clasmatodendrose und Sektionszeit angestellt. Das Ergebnis ist in nebenstehender Tabelle 1 graphisch dargestellt. In der Abszisse ist die vom Augenblick des Todes bis zur Sektion verstrichene Zeit in Stunden eingetragen (darunter die Zahl der untersuchten Fälle), während auf der Koordinate die Prozentzahlen der positiven Fälle abgelesen werden können.

Aus der Tabelle ergibt sich eine erhebliche Zunahme der Fälle mit Gliazerfall innerhalb der ersten 10 Stunden von $41,2 \%$ auf $75 \%$, während der Unterschied später wesentlich geringer wird und nach Ablauf von 20 Stunden als 0 bezeichnet werden kann. Dies Ergebnis scheint auf den ersten Blick in einem direkten Widerspruch mit Wohlwills Befunden zu stehen. In Wirklichkeit besteht dieser aber nicht, da unter 
seinen 126 Fällen nur ein einziger vor Ablauf der 10 Stunden seziert ist, so da $\beta$ ein analoges Verhältnis hier gar nicht zum Ausdruck kommen konnte. Im übrigen sind die beiden Statistiken bezüglich ihrer Prozentzahlen wegen der verschiedenen benutzten Methoden und des ganz anders gearteten Materials kaum vergleichbar.

Nun sind freilich die positiven Fälle der nebenstehenden Tabelle keineswegs als gleichartig anzusehen, da sich unter ihnen alle Grade von eben beginnender Clasmatodendrose bis zum fast völligen Zerfall aller plasmatischen Gliazellen finden. 'Ich habe deshalb versucht, diese weiter zu differenzieren und die Schwere des Zerfalls durch die verschieden schraffierten Felder angedeutet. Dabei ist allerdings zu bemerken, da $\beta$ ein subjektives Moment nicht $\mathrm{zu}$ umgehen ist, da eine exakte quantitative Unterscheidung nicht möglich ist. Immerhin gibt die Tabelle doch ein ungefähres Bild der Verteilung. Zunächst fällt auf, daß in der ersten Rubrik ein gewisser Prozentsatz ganz schwerer Fälle vorhanden ist, der in der zweiten und dritten fehlt, und daß dieser in der letzten Rubrik wiederum etwas kleiner ist als in der vorletzten, und hier überhaupt auffallend viele Fälle mit geringem Zerfall vorhanden sind. Hier spielen gewisse Zufälligkeiten eine Rolle. Naturgemäß wären die stark positiven Fälle der ersten Rubrik, wenn sie zu-

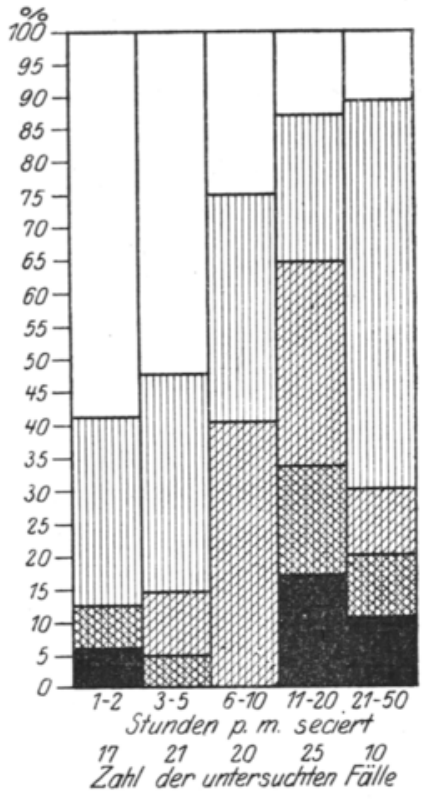

Fast völliger Zerfall der plasmatischen Glia.

838\% StarkerZerfall, nurin 6.Rindenschicht oder oberster Markleiste erhaltene Spinnenzellen.

MäBiger Gliazerfall.

Leichter Zerfall.

Gut erhaltene Glia. fällig später seziert wären, in einer der folgenden wieder erschienen. Sie beweisen also nur, daß schwerer Gliazerfall auch schon sehr früh nach dem Tode vorhanden sein kann. Zur Erklärung der großen Zahl leichter Fälle unter den nach der 20. Stunde sezierten Gehirnen kommt aber noch ein andres Moment von allgemeinerer Bedeutung in Frage. Es befinden sich nämlich 6 Gehirne (also $60 \%$ ) von senil Dementen darunter. Ich habe nun immer wieder beobachten können, daß hypertrophische Gliazellen widerstandsfähiger sind als solche normaler Struktur, gleichgültig, ob es sich um reine Volumzunahme handelt, wie sie vor allem bei entzündlichen Prozessen beobachtet wird, oder um die besonders bei Senilen auftretenden hageren Zellen mit langen stark 
verzweigten Dendriten. Besonders deutlich wird dieses differente Verhalten dort, wo hypertrophische Astrocyten neben normalen vorkommen! So konnte ich mehrere Fälle von Meningitis untersuchen, wo die stark hypertrophische Randglia noch völlig intakt war, während in den darunter liegenden Schichten bereits fortgeschrittener Zerfall eingetreten war. Auch in der senilen Hirnrinde finden sich fast regelmäßig einzelne oder Gruppen stark hypertrophischer Zellen, die als einzige von Zerfall verschont bleiben können, während sich in der unmittelbaren Umgebung mehr oder weniger deutliche Clasmatodendrose beobachten läßt. Da auch die Markglia bei seniler Demenz meist eine Veränderung in dem erwähnten Sinne zeigt, sind hier die Differenzen zwischen früh und spät sezierten Fällen bezüglich der Clasmatodendrose nur gering, und bei Paralyse beziehen sie sich vorwiegend auf das Marklager, wo die Gliahypertrophie meist wesentlich geringer ist als in der Rinde. Nie habe ich bei seniler Demenz oder der Paralyse einen so völligen Zerfall gesehen, wie z. B. bei Dementia praecox oder funktionellen Psychosen.

Berücksichtigt man diese Tatsachen, so lösen sich damit die scheinbaren Widersprüche der Tabelle, und man wird sie dann nur in dem Sinne deuten können, daß mit der Veriängerung der Zeit vom Tode bis zur Fixierung des Gehirnes - besonders in den ersten 10 Stunden die Zahl der positiven Fälle zunimmt.

Es lag nahe, die mutmaßliche postmortale Autolyse der Glia mit der gleichen Methode auch am einzelnen Gehirn zu verfolgen. Dabei war ich mir aber von vornherein darüber klar, daß es mehr oder weniger unmöglich ist, worauf besonders auch Wohlwill hingewiesen hat, natürliche Verhältnisse zu schaffen. Bei den noch ganz unklaren physikalischen und chemischen postmortalen Verhältnissen in der Schädelkapsel besagen Beobachtungen an zu verschiedenen Zeiten excidierten Stückchen, die in Liquor oder sonstigen Flüssigkeiten aufgehoben werden, nicht sehr viel. Mir schien es den natürlichen Verhältnissen am nächsten zu kommen, wenn man möglichst frühzeitig p. m. mit Hilfe einer Trepanationsöffnung ein Gehirnstückchen excidierte, den Schädel wieder schloß, um nach längerer Zeit das Gehirn in toto zu sezieren und dieses Obduktionsmaterial mit dem ersten Stück zu vergleichen. Von 4 derartig untersuchten Fällen (Trepanation innerhalb der ersten 2 Stunden, Sektion nach Ablauf von mindestens 20 Stunden) zeigte sich einmal ein deutlicher, einmal ein zweifelhafter, zweimal kein Unterschied! Ob resp. wieweit bei dieser Versuchsanordnung die durch die Trepanation veränderten Druckverhältnisse in der Schädelkapsel, der Luftzutritt und Liquorabfluß eine Rolle spielen, muß ich dahingestellt bleiben lassen.

Bereits Cajal hat betont, daß auch schlechte Fixierung Autolyse 
der Glia hervorrufen kann, was den Rosentalschen Befunden einer postmortalen amöboiden Umwandlung in verschiedene Flüssigkeiten entspricht. Zur Beantwortung der Frage habe ich in 2 Fällen möglichst gleich große Gehirnstückchen in 1 proz., 5 proz., 20 proz. und 50 proz. Formalin gehärtet und in mehreren Fällen das in reichlicher 14 proz. Brom-Formol-Lösung fixierte Stückchen mit einem in 10 proz. Formol fixierten Gehirn entnommenen verglichen und schließlich einen Teil des Materials in sehr häufig gewechselten Lösungen, einen Teil in der gleichen Lösung durchfixiert. Die beiden letzten Versuchsreihen fielen negativ aus, dagegen zeigten Präparate aus 5 proz. Formalin an den Spinnenzellen der Rinde Varicositäten und zum Teil Diskontinuität der Dendriten, während im Mark eine Differenz nicht deutlich war. Das Material aus 1 proz. Formalin färbte sich ungenügend. Besonders im Mark waren die Dendriten nur kurze Strecken imprägniert, aber nicht zerfallen. Die Versuchsreihe muß erst noch vervollständigt werden durch Untersuchung an möglichst verschiedenem Material, denn ebenso wie sich die Gehime unter normalen Fixationsbedingungen verschieden verhalten, könnte dies auch hierbei der Fall sein. Immerhin erscheint die Warnung Cajals schon nach dem bisherigen Ergebnis durchaus berechtigt. Erwähnen möchte ich noch, daß altes Formolmaterial keine guten Resultate bei der Gold-Sublimatmethode gibt. Vor allem bekommt man fast nie brauchbare Rindenbilder. Oft sind die vorhandenen Dendriten grobkörnig inkrustiert, so daß auf den ersten Blick eine Verwechslung mit beginnender Clasmatodendrose wohl möglich ist. Auch im Zwischengewebe sieht man nicht selten schwarzgefärbte Körner, die Gliadetritus ähneln.

Untersucht man Gehirne mit den beiden angewandten Methoden systematisch durch, so stößt man sehr bald auf gewisse Gesetzmäßigkeiten bezüglich der Ausbreitung des Gliazerfalls, die m. E. nur durch weitgehende Unabhängigkeit von den jeweiligen individuellen Verhältnissen - Art der psychischen und körperlichen Erkrankung, Alter der Person usw. - erklärt werden können und wohl auf gleichartige physikalische und chemische Faktoren im Gehirn zurückgeführt werden müssen.

Schaffer hat behauptet, die Cajalsche Methode eigne sich nicht zur Darstellung der normalen Glia der oberen Rindenschichten. Das ist nach meinen Erfahrungen nicht richtig! Wo die Färbung dieser Schichten ausbleibt - natürlich bei sonst gut gelungener Imprägnation - ist die Ursache dafür entweder in zu langer Fixation oder dem bereits eingetretenen Zerfall der zarten Astrocyten daselbst zu suchen. Einzelne größere Spinnenzellen färben sich in den meisten Fällen auch in den obersten drei Rindenschichten, aber eine gute und scheinbar vollständige Darstellung der plasmatischen Glia dieser Zone habe ich 
bisher nur in ganz früh sezierten Fällen gesehen. Meist fanden sich auch hier schon an den Dendriten besonders der zweiten Schicht grobe Körnelung und beginnende Diskontinuität, die ich zum Teil als Anfang der Clasmatodendrose ansehen möchte. Freilich mit einigem Vorbehalt, da ich zuweilen bei ganz zarter Imprägnation in einem Präparat feinwabige, aber kontinuierliche Fortsätze sah, während ein stärker gefärbtes die erwähnten Veränderungen zeigte. (In obiger Tabelle sind diese Fälle als negativ bezeichnet.) Nach der Tiefe zu, besonders in der 5. und 6. Schicht, sind die Astrocyten normalerweise größer und besitzen offenbar auch eine dichtere Plasmastruktur, worauf wohl die stärkere und leichtere Tinktion mit fast allen Methoden beruht. Damit ist aber zugleich eine höhere Widerstandskraft gegen Zerfall verbunden. Auch in den Fällen mit extremster Clasmatodendrose habe ich hier immer wenigstens noch einige intakte Astrocyten gefunden. Das gleiche gilt für die oberste Markleiste, während im eigentlichen Mark der Gliazerfall mit größter Regelmäßigkeit nach der Tiefe zunimmt, so daß man hier schon völlige Zerstückelung der Dendriten in Fällen findet, wo die oberflächlichen Partien erst den Beginn derselben zeigen. Bei dieser Gelegenheit möchte ich bemerken, daß die pulverförmige Verteilung des Gliadetritus, gegenüber der reihenweisen Anordnung der Fortsatzfragmente kein differentialdiagnostisches Merkmal zwischen vitaler und postmortaler Ciasmatodendrose darstellen kann, sondern lediglich Ausdruck weit fortgeschrittenen Zerfalls ist. Nur so ist es erklärlich, daß man je nach dem Ort beide im gleichen Gehirn nebeneinander findet. - In den Stammganglien und der inneren Kapsel ist der Gliazerfall meist geringer als im tiefen Zentralmark.

Ohne Ausnahme fand ich das geschilderte Verhalten bis jetzt bezüglich der Resistenzfähigkeit der Glia der 6. Schicht und die zunehmende Zerfallstendenz nach der Tiefe des Marklagers zu, während von dem primären Zerfall der oberflächlichen Rindenglia, wenn auch seltener Ausnahmen vorkommen, ohne daß eine Hypertrophie hier nachweisbar ist. Ebenso verhält sich die plasmatische Glia des Striatums und der inneren Kapsel etwas verschieden. Die erwähnte Gesetzmäßigkeit findet sich, abgesehen von quantitativen Unterschieden im ganzen Gehirn. Am frühesten und stärksten scheint die Clasmatodendrose im allgemeinen im tiefen Parietalmark aufzutreten.

Die wichtigste Frage bleibt naturgemäß die, ob und wieweit diffuser Gliazerfall eine -- direkte oder indirekte - Abhängigkeit von bestimmten körperlichen und psychischen Krankheitszuständen zeigt. Anfangs glaubte ich, daß die endogenen Verb'ödungen eine besondere Tendenz dazu aufwiesen, weil mir als Vergleichsobjekte besonders Paralysen und senile Erkrankungen zur Verfügung standen, und ich die erhöhte Resistenzfähigkeit der hyperplastischen Glia noch nicht kannte. Als 
ich dann aber auch mehrere Fälle von funktionellen Psychosen zu untersuchen Gelegenheit hatte, mußte ich diesen Gedanken aufgeben. Nach dem jetzt immerhin schon recht großen Vergleichsmaterial halte ich es für unmöglich, solche direkten Kausalbeziehungen anzunehmen. Auch der akute oder chronische Verlauf einer Psychose hat nach meinen Erfahrungen keine wesentliche Bedeutung für das Auftreten der Clasmatodendrose. Eisath glaubte ja, daß besonders die in akuten Phasen der Dementia praecox Verstorbenen amöboide Umwandlung zeigten, und auch Alzheimer nahm ähnliche Zusammenhänge an. Viele von meinen in exquisit chronischen Phasen der Psychose an Tuberkulose, Pneumonie, Dysenterie usw. verstorbenen Kranken zeigten jedoch hochgradigen Zerfall der Glia und umgekehrt, allerdings $m_{*} i$ wenigen Ausnahmen. Die beiden bisher einzigen Fälle, die trotz unmittelbar (1 und $1 \frac{1}{2}$ Stunden) post mortem vorgenommener Sektion starke Clasmatodendrose aufwiesen, betrafen schwere Erregungszustände mit starker Hyperkinese, nämlich eine Chorea infectiosa gravis und ein Delirium acutum unklarer Ätio'ogie. Wenn auch die Zahl dieser Beobachtungen zu gering ist, um eine definitive Entscheidung zu fällen, so spricht doch die Tatsache fär einen wie auch immer gearteten $\mathrm{Zu}$ sammenhang! Aber auch bei dieser Annahme ist die Frage noch nicht entschieden, ob der Gliazerfall auf vitalen oder postmortalen Vorgängen beruht. Ich kann mich voriäufig nicht zu der ersten Alternative entschließen, und zwar aus folgenden Gründen: Lot mar hat nachgewiesen, daß Körnchenzellabbau und amöboide Umwandlung der Glia zwei einander nahezu ausschließende Vorgänge sind, und daß letztere nur dort vorkommt, wo es sich um einen perakuten Prozeß handelt und ein Abbau der Zerfallsstoffe durch die Glia nicht mehr möglich ist. Nach der heutigen, wohlbegründeten Anschauung ist ferner die plasmatische Glia ein für das Leben und die Funktion der nervösen Organe außerordentlich wichtiges Element. Beides verträgt sich aber kaum mit der Annahme, daß ein diffuser Gliazerfall im ganzen Gehirn mit dem Leben auch nur für kürzere Zeit vereinbar sei. Schließlich wäre es auch, bei der Voraussetzung eines vitalen Prozesses, unerklärlich, daß häufig z. B. bei funktionellen Psychosen die nervösen Elemente völlig intakt gefunden werden können. Auch wenn man die Clasmatodendrose als primären Vorgang ansehen wollte, müßte sie doch notwendig in kürzester Zeit zu schweren Veränderungen auch der Nervenzellen führen.

Ich glaube also, daß auch bei diesen schweren Erregungszuständen der Gliazerfall de facto erst im Augenb'ick des Er'öschens der nervösen Funktionen einsetzt, während der vitale Krankheitsprozeß im wesentlichen die Bedingungen schafft, die zum Zerfall führen.

Die Bedeutung des prämortalen Gehirnzustandes für das Auftreten der amöboiden Umwandlung ist ja von fast allen Untersuchern be- 
hauptet. Rosentals Beobachtung, daß bei vergifteten und überhitzten Tieren schon $2-3$ resp. 5-6 Stunden p. m. schwere autolytische Veränderungen vorhanden sein können, während solche bei normalen Tieren erst später auftreten, sind ja kaum anders zu erklären, ohne daß damit freilich eine bestimmte Ursache gegeben ist. Wohlwill sieht in den Zirkulationsstörungen, speziell dem Gehirnödem den entscheidenden Faktor. Wenn ich auch von der Wichtigkeit des letzteren fest überzeugt bin, so liegen die Verhältnisse doch nicht so, daß hiermit allein alles erklärt wäre, denn ich finde unter meinem Material eine Reihe von Beispielen, wo trotz weitgehendem Zerfall ein nachweisbares Gehirnödem fehlte und umgekehrt. Ich will nur wenige Beispiele anführen:

1. (Fall 21 der obenangeführten) Diagnose. Cystischer Hypophysentumor mit Übergreifen in Frontalhirn. Sektion $1 / 4$ St. p. m. Starkes Piaödem, starke Hyperämie des ganzen Gehirns und starker Feuchtigkeitsgehalt. Histologisch: Keine Clasmatodendrose, keine amöboide Glia (abgesehen von unmittelbarer Umgebung des Tumors).

2. H., 32 Jahre, 우. Schwerer halluzinatorischer Verwirrtheitszustand. Gest. an Miliartuberkulose. Sektion 26 St. p. m. Starkes Piaödem, starke Hyperämie und Feuchtigkeitsgehalt des Gehirns. Histologisch: Kein Gliazerfall.

3 v. 0., 64 Jahre, ơ. Katatonie. Gest. an Dysenterie. Sektion $4^{1 / 2}$ St. p. m. Kein Piaödem, keine Hyperämie, keine abnorme Feuchtigkeit des Gehirns. Histologisch: Starke allgemeine Clasmatodendrose, reichliche amöboide Gliazellen.

Wenn es sich hierbei auch wohl um Ausnahmefälle handelt, so zeigen sie doch, daß die Verhältnisse nicht einfach liegen. Man könnte nun freilich einwenden, daß Piaödem, allgemeine Hyperämie und vermehrter Feuchtigkeitsgehalt des Gehirns noch kein Beweis für eine Hirnschwellung sind. Aber Wohlwill betont extra, daß er diesen Begriff nicht im engen Sinne Reichardts verstanden wissen will, und an Zirkulationsstörungen jedweder Art denkt, wofür Hyperämie und vermehrte freie Flüssigkeit Anzeichen sind. Aber gerade von diesem meines Erachtens ganz richtigen Standpunkt aus gewinnen die angeführten Fälle ihre Bedeutung. Allerdings ist dabei zu berücksichtigen, daß die bei der Sektion gefundenen Verhältnisse noch nicht ohne weiteres auf den lebenden Organismus übertragen werden dürfen. Der Flüssigkeitsgehalt des Gehirns bleibt post mortem wohl sicherlich nicht konstant, sondern es wird der im Subarachnoidalraum vorhandene Liquor unter Umständen vom Gehirn resorbiert werden können. So erkläre ich mir wenigstens die Tatsache, daß die Menge des Liquors, wenn man längere Zeit nach dem Tode punktiert, zuweilen auffallend gering sein kann. Aber trotz aller dieser Bedenken halte auch ich ein intravital entstandenes Gehirnödem für einen wichtigen Faktor.

Schon Alzheimer wies auf das Vorkommen amöboider Zellen in der Umgebung von Tumoren, Apoplexien usw. hin, und Wohlwill 
führt ja mehrere Beispiele dafür in seiner zitierten Arbeit an. Ich will nur einen Fall kurz erwähnen, der dasselbe bezüglich der Clasmatodendrose zeigt, aber noch in andrer Hinsicht von Interesse ist.

J., 52 Jahre, ơ. Stirnhirngliom r. Plötzlicher Exitus. Sektion 6 St. p. m. Pia stark injiziert. Windungen beiderseits, aber $\mathbf{r}$. deutlich mehr als 1. abgeplattet. Rechte Hämisphäre besonders im Vorderteil aufgetrieben. Mediale Fläche nach links verschoben. Frontalschnitt durch vordersten Teil des Balkens zeigt rechts ca. walnußgroßen Tumor, der hier ziemlich scharf gegen Umgebung abgegrenzt ist. Marklagen der Hemisphäre stark verbreitert und außerordentlich weich und feucht, aber nicht zerfallen. Es wird rechts ein bis an die Oberfläche reichendes Rechteck aus der unmittelbaren Umgebung des Tumors zur Untersuchung herausgenommen, ebenso links von der analogen Stelle. Histologischer Befund. Rechts fortgeschrittener Zerfall der Glia, von dem nur einzelne stark hypertrophische Zellen (ob es sich um Gliomzellen oder reaktiv hypertrophische Zellen handelt, ließ sich nicht sicher entscheiden) verschont geblieben sind. Links ist die Glia gut erhalten. Nur vereinzelt sind Körner und Bröckel im Zwischengewebe sichtbar.

Der Fall zeigt einmal, daß starkes Hirnödem auch hypertrophische, d.h. also sehr widerstandsfähige Gliazellen zum Zerfall bringen kann, dann aber auch, daß relativ starkes Hirnödem, wie es links vorhanden war, sogar gegenüber normalen Zellen unwirksam bleiben kann.

Ähnliches habe ich in mehreren anderen Fällen gesehen., Wir werden aus alledem den Schluß ziehen müssen, daß nicht ein Faktor ausschlaggebend ist, sondern daß solche verschiedenster Art wirksam sein können. Dabei ist neben physikalischen Verhältnissen, besonders an rein chemische resp. toxische Schädigungen zu denken, mögen diese nun endogener oder exogener Art sein. Jedenfalls müssen auch alle körperlichen Erkrankungen, die keine manifesten psychischen Symptome hervorrufen, in dieser Hinsicht berücksichtigt werden. Meine hierauf gerichteten Untersuchungen haben aber zu keinem greifbaren Resultat geführt: So sah ich z. B. bei schwerer Tuberkulose oder Dysenterie bald ausgesprochenen Gliazerfall, bald keinen und ebenso bei den meisten andern Erkrankungen. Nur bei allgemeiner Sepsis fand ich bisher (3 Fälle) stets reichliche Clasmatodendrose, obwohl ein Fall bereits $4^{1} / 2$ Stunden p. m. seziert werden konnte. Aber es fehlen noch ganz frühe Sektionen, bevor etwas Sicheres gesagt werden kann. Auch die Körpertemperatur, die kurz vor dem Tode bestanden hat, ist nicht ausschlaggebend für das Verhalten der Glia! Ich brauche in dieser Beziehung nur auf die oben einzeln kurz angeführten Fälle zu verweisen.

In den obigen Ausführungen ist stets von der Clasmatodendrose als diffusem Gehirnprozeß die Rede gewesen, deren intravitale Entstehung sehr unwahrscheinlich ist. Damit ist natürlich nicht gesagt, daß jeder Gliazerfall dieser Art so zu deuten ist. Ich bin im Gegenteil überzeugt, daß in allen circumscripten Herden mit nicht reparablen Funktionsstörungen der nervösen und gliösen Elemente auch intra 
vitam der gleiche Prozeß vor sich geht. Aber der morphologische Verlauf der Clasmatodendrose weist meines Erachtens doch an sich schon darauf hin, daß hier kein Zellabbau, sondern eine Nekrose vorliegt. Wenn der Herd an nicht lebenswichtiger Stelle liegt, und es tritt Vernarbung ein, dann müssen andere Elemente vor allem die Körnchenzellen die reparatorische Funktion übernehmen.

Nun findet man freilich, worauf schon Cajal, Achucarro und Schaffer aufmerksam gemacht haben, nicht selten einzelne in Zerfall begriffene Zellen zwischen lauter normalen. Daß es sich dabei nicht immer um den Beginn einer allgemeinen Clasmatodendrose zu handeln braucht, schließe ich daraus, daß solche untergehenden Elemente auch in Zonen zu finden sind, z. B. der obersten Markleiste und unterster Rinde, die nach unsern obigen Mitteilungen am widerstandsfähigsten sind, während das tiefe Mark und die obere Rindenhälfte noch mehr oder weniger intakt sind. Auch können zweifellos hypertrophische Zellen isoliert befallen werden. Die Tatsache, daß solche Beobachtungen auch an völlig ,,normalen" Gehirnen gemacht werden können, legt den Gedanken nahe, ob es sich nicht um ein physiologisches Absterben, also eine Alterserscheinung der Zelle handeln kann. Doch werden hier weitere Untersuchungen notwendig sein.

Fasse ich das Ergebnis der Arbeit nochmals kurz zusammen, so möchte ich folgendes sagen:

Die Clasmatodendrose Cajals ist prinzipiell der gleiche Vorgang wie die amöboide Umwandlung Alzheimers.

Sie kommt als diffuser Prozeß häufiger vor, als nach Untersuchungen mit den Alzheimerschen Methoden zu erkennen ist.

Häufigkeit und "Umfang der Clasmatodendrose nehmen besonders in den ersten 10 Stunden post mortem zu. Auch schlechte Fixierung des Gewebes kann zu gleichen autolytischen Veränderungen führen.

Der Gliazerfall zeigt eine gewisse Gesetzmäßigkeit insofern, als die plasmatische Glia der obersten Rindenschichten am frühesten zerfällt, während 5. bis 6 . Schicht und oberste Markleiste die widerstandsfähigsten Astrocyten enthält.

Im Mark ist eine Zunahme der Clasmatodendrose nach der Tiefe zu die Regel.

Unabhängig von aller Lokalisation gilt aber, daß hypertrophische Gliazellen resistenter sind als normale.

Der diffuse Gliazerfall ist als autolytischer agonaler oder postmortaler Prozeß aufzufassen. Ein direkter Zusammenhang mit Gehirnkrankheiten und Psychosen ist nicht nachweisbar. Doch spielt der prämortale Gehirnzustand insofern eine Rolle, als starkes Gehirnödem den agonalen oder postmortalen Zerfall fördert.

An circumscripten Stellen kommt die Clasmatodendrose infolge 
schwerer akuter Schädigung intra vitam vor und ist hier als eine Form der G ianekrose aufzufassen.

Außerdem wird Zerfall einzelner, plasmatischer Gliazellen beobachtet bei Fehlen aller sonstigen pathologischen Erscheinungen. Möglicherweise handelt es sich dabei um physiologische Alterserscheinungen der Zellen. Ein Beweis für eine Gehirnerkrankung kann aus dem Vorhandensein diffusen Gliazerfalls nicht abgeleitet werden.

\section{Literaturverzeichnis.}

Achucarro, N. y M. Gayarre, La corteza cerebral en la demencia paralítica con el nuovo método del oro y sublimado de Cajal. Trabajos del Labor. d. Investigac. biol. d. l. Univers. Madrid 12. 1914. - Alzhei mer, A., Beiträge zur Kenntnis d. pathol. Neuroglia usw. Histologische u. histopathologische Arbeiten 3. - Cajal, R., Contribución al concimento de la neuroglia del cerebro humano. Trab. d. Lab. d. Invest. biol. d. 1. Univ. Madrid 11. 1914. Ref. Diese Zeitschr. 10. 109. - Buscai no, M. , Sulla genesi e sub significato d. cellul ameboidei. Revist. d. Pathol. nerv. et ment. 18, 360. 1913. Ref. Diese Zeitschr. 8, 83. - Eisath, Weitere Beobachtungen über das menschliche Nervenstützgewebe. Arch. f. Psych. 48. 1911. - Eisath, Über normale und pathologische Histologie der menschlichen Glia. Monatsschr. f. Psych. u. Neurol. 20. - Lot ma r, Fr., Beiträge zur Kenntnis der akuten Myelitis und Encephalitis, sowie verwandter Prozesse. Histol. u. histopathol. Arbeiten 6, 245. 1913. - Rosental, Experimente über amöboide Umwandlung der Neuroglia. Histolog. u. histopathol. Arbeiten 6, 89. 1913. - Wohl . will, Fr., Über amöboide Glia. Virchows Archiv 216, 468. 1914. — Wa tter, F. K., Zur normalen und pathologischen Histologie der Neuroglia. Sitzungsber. d. Naturf. Gesellsch. z. Rostock v. 5. VII. 1918. - Walter, F. K., Beiträge zur Histopathologie der endogenen Verblödungen. Diese Zeitschr. 4\%, 112. 1919.

Nachtrag bei der Korrektur: In letzter Zeit habe ich eine Reihe von Gehirnen zur Fixierung möglichst frisch mit warmer Formollösung injiziert. Unter diesen habe ich bisher keinen Fall mit stärkerem Gliazerfall gefunden, was entschieden für die Wichtigkeit der Fixierung spricht. 